\title{
Thermo-optic Characteristics of Micro-structured Optical Fiber Infiltrated with Mixture Liquids
}

\author{
Ran Wang ${ }^{1,2}$, Yuye Wang ${ }^{1, *}$, Yinping Miao ${ }^{1,2,3}$, Ying $\mathrm{Lu}^{1,2}$, Nannan Luan ${ }^{1,2}$, Congjing Hao ${ }^{1,2}$, \\ Liangcheng Duan ${ }^{1,2}$, Cai Yuan, ${ }^{1,2}$ and Jianquan Yao ${ }^{1,2}$ \\ ${ }^{1}$ College of Precision Instruments and Opto-electronics Engineering, Institute of Laser \& \\ Optoelectronics, Tianjin University, Tianjin 300072, China \\ ${ }^{2}$ Key Laboratory of Opto-electronics Information Technology (Tianjin University), Ministry of \\ Education, Tianjin 300072, China \\ ${ }^{3}$ School of Electronics Information Engineering, Tianjin Key Laboratory of Film Electronic and \\ Communication Device, Tianjin University of Technology, Tianjin 300384, China
}

(Received January 21, 2013 : revised April 15, 2013 : accepted April 15, 2013)

\begin{abstract}
We present both theoretically and experimentally the thermo-optic characteristics of micro-structured optical fiber (MOF) filled with mixed liquid. The performance of MOF depends on the efficient interaction between the fundamental mode of the transmitted light wave and the tunable thermo-optic materials in the cladding. The numerical simulation indicates that the confinement loss of MOF presents higher temperature dependence with higher air-filling ratios $d / \Lambda$, longer incident wavelength and fewer air holes in the cladding. For the $4 \mathrm{~cm}$ liquid-filled grapefruit $\mathrm{MOF}$, we demonstrate from experiments that different proportions of solutions lead to tunable temperature sensitive ranges. The insertion loss and the extinction ratio are 3 4 $\mathrm{dB}$ and approximate $20 \mathrm{~dB}$, respectively. The proposed liquid-filling MOF will be developed as thermo-optic sensor, attenuator or optical switch with the advantages of simple structure, compact configuration and easy fabrication.
\end{abstract}

Keywords: Micro-structured optical fiber, Thermo-optic characteristics, Mixture liquid, Thermo-optic sensor, Optical switch

OCIS codes : (060.4005) Microstructured fibers; (230.1150) All-optical devices; (280.4788) Optical sensing and sensors; (130.4815) Optical-switching devices

\section{INTRODUCTION}

Micro-structured optical fibers (MOFs) [1-2] have attracted considerable research interest in recent years due to their novel light guidance properties [3-4]. The micrometer- sized air holes running along the length of the fiber provide great freedom in optical waveguide design during the fabrication procedure [5-6]. Additionally, the infiltration of functional materials into claddings provides further responses under different external physical fields, leading to tunable propagation properties of MOFs for promising all-in-fiber optical components [7-8].

Compared with the mechanical counterpart, the waveguide devices based on liquid-filling MOFs [9] provide efficient control and modification of optical signals for the development of optical communication and sensing systems [10], leading to the advantages of low insertion loss, high sensitivity, performance stability and integrity. T. Larsen [11] first demonstrated a low-voltage controlled broadband optical switch infiltrated with liquid crystals, with the extinction ratio as much as $60 \mathrm{~dB}$ for a $0.4^{\circ} \mathrm{C}$ temperature interval and insertion loss of $1 \mathrm{~dB}$. Y. Yu and $\mathrm{X}$. Li introduced a novel MOF temperature sensor based on liquid ethanol infiltrated into the cladding with the sensitivity of 0.315 $\mathrm{dB} /{ }^{\circ} \mathrm{C}$ [12]. Optical switches have been reported by $\mathrm{Y}$. Wang and W. Jin based on fluid-filled MOF Bragg grating [13] or temperature-controlled hybrid micro-structured fiber [14], with the extinction ratio $>30 \mathrm{~dB}$ via the temperature adjustment of $\pm 5^{\circ} \mathrm{C}$. They also investigated a thermo-optical switch based on the absorption of the filled fluid in

\footnotetext{
*Corresponding author: yuyewang@tju.edu.cn

Color versions of one or more of the figures in this paper are available online.
} 
combination with the interaction between the core mode and the excited "fluid rod" modes [15]. According to all the reports above, the cross section structure of MOFs is significant for the fabrication of optical components and needs systematical analysis with numerical simulation. Additionally, the temperature sensitivity range of the components is always constant and cannot be tunable in response to the exotic environment, which severely limits their practical application.

In this letter, we have demonstrated the thermo-optic characteristics of liquid-filling solid core MOFs, which exploits temperature dependence on the refractive index of mixture liquids incorporated into the cladding. Numerical simulation illustrates that the greater air-filling ratio, longer incident wavelength, and fewer air holes in the cladding could effectively enhance the performance of the device. For a 4-cm-long liquid-filling grapefruit MOF, with the different concentration of the two components (toluene and chloroform), it presents a tunable high temperature-sensitive range within $5^{\circ} \mathrm{C}$. The insertion loss is about $3 \sim 4 \mathrm{~dB}$ and the extinction ratio is approximate $20 \mathrm{~dB}$, respectively. As an intensitymodulated optical component, expensive and high precision apparatus such as broadband amplified spontaneous emission (ASE) fiber source and high-resolution optical spectrum analyzer (OSA) are not necessary. Such a device will be developed as a thermo-optic sensor, attenuator or slow response optical switch with the advantages of simple structure, high integration and easy fabrication with the lowest cost.

\section{THEORETICAL ANALYSIS AND NUMERICAL SIMULATION}

The interaction between the evanescent field and the cladding air voids makes the core mode very sensitive to any refractive index fluctuation at the air-hole-cladding interface (the boundary between the air holes in the cladding and the solid fiber core, as indicated in Fig. 1). Tunable

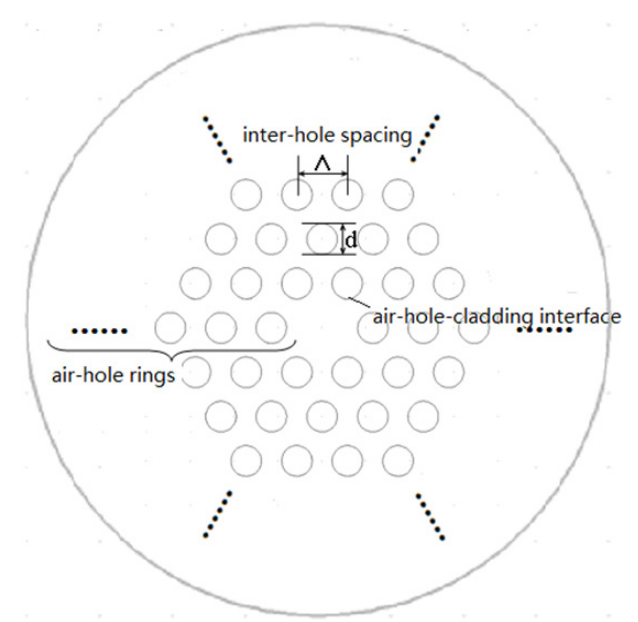

FIG. 1. The cross-section and the structure parameters of MOFs with identical cladding configuration for FEM simulation. refractive index materials, such as temperature-sensitive mixture liquids (toluene and chloroform), could be introduced into the air holes and will modulate the guiding properties of MOFs. For the temperature behavior of the refractive index of the liquids, a linear approximate expression

$$
n(\Delta T)=n_{0}+\frac{d n}{d T} \cdot \Delta T
$$

has been assumed, in which $n_{0}$ denotes the refractive index from the Sellmeier equation. $\Delta T$ is the difference between the absolute temperature $T$ and the temperature $T_{0}$ at which the Sellmeier coefficients are given. The thermo-optical coefficients $d n / d T$ amount to $-5.273 \times 10^{-4} / \mathrm{K}$ for toluene and $-6.328 \times 10^{-4} / \mathrm{K}$ for chloroform [16]. Furthermore, the thermooptical coefficients are assumed independent with the incident wavelength and temperature. In contrast to the value of the liquids, the thermo-optical coefficient of fused silica $\left(8.6 \times 10^{-6} / \mathrm{K}\right)$ is two orders of magnitude lower than that of liquids and is therefore not taken into consideration. In order to obtain the refractive index of the liquid mixtures, the Lorentz-Lorenz equation is used [17]:

$$
\frac{n^{2}-1}{n^{2}+2}=\phi_{1} \frac{n_{1}^{2}-1}{n_{1}^{2}+2}+\phi_{2} \frac{n_{2}^{2}-1}{n_{2}^{2}+2}
$$

Here, $n_{1} n_{1}$, and $n_{2}$ are the refractive index of the solution and the constituents, respectively. $\Phi_{1}$ and $\Phi_{2}$ are the volume fractions of the constituents and $\Phi_{2}$ can be replaced by $1-\Phi_{1}$.

To investigate the dependence of the propagation characteristics on the structure parameters, a series of MOFs with identical cladding configuration and inter-hole spacing (which means the distance between the centers of the adjacent air holes, taking $\Lambda=6$ um in our simulation) have been simulated under different air-filling ratios, air-hole rings in the cladding and incident wavelengths. The air-filling ratio is defined as $d / \Lambda$ and denotes the proportion of the air holes in the cladding while the air-hole ring means the number of the air holes. The core is a defect of 1-ring air holes and the cladding is two-dimensional (2D) hexagonal arrangement holes, as shown in Fig. 1. Taking the volume ratio of toluene and chloroform as 7:3 for example, the confinement loss properties of the liquid-filled MOFs are numerically investigated based on the full-vector finite element commercial software packages COMSOL Multiphysics combined with the anisotropic Perfectly Matched Layer (PML). The confinement loss $(\mathrm{dB} / \mathrm{m})$, arising from the imaginary part of the effective complex refractive index $n e_{f f}$, is given as an expression [12]:

$$
C L(d B / m)=20 \log _{10} e \times \operatorname{Im}\left(\beta_{e f f}\right)=8.686 \times \operatorname{Im}\left(n_{e f f}\right) \times k_{0}
$$

The results are shown in Fig. 2 in which the confinement losses are illustrated against the temperature with the air- 


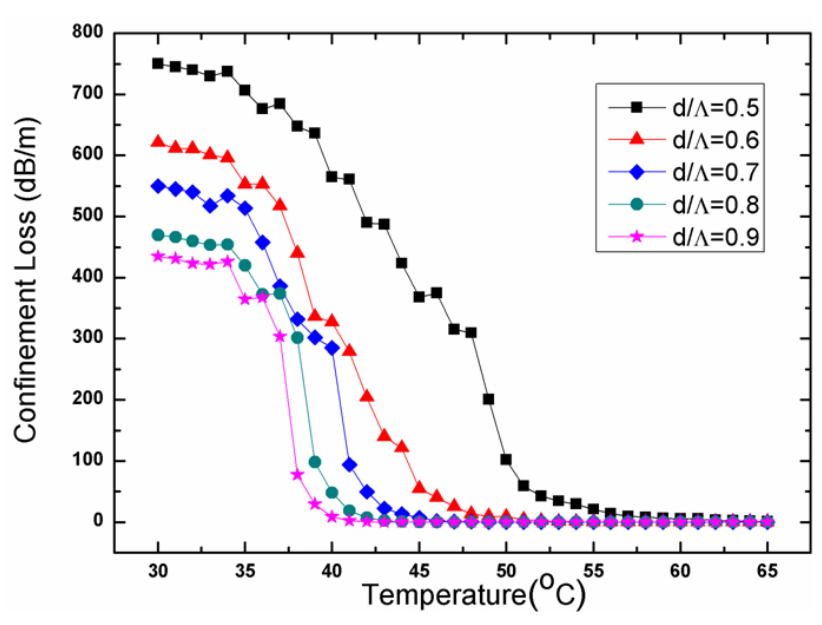

FIG. 2. Confinement loss as functions of temperature with different air-filling ratios $d / \Lambda=0.5,0.6,0.7,0.8,0.9$.

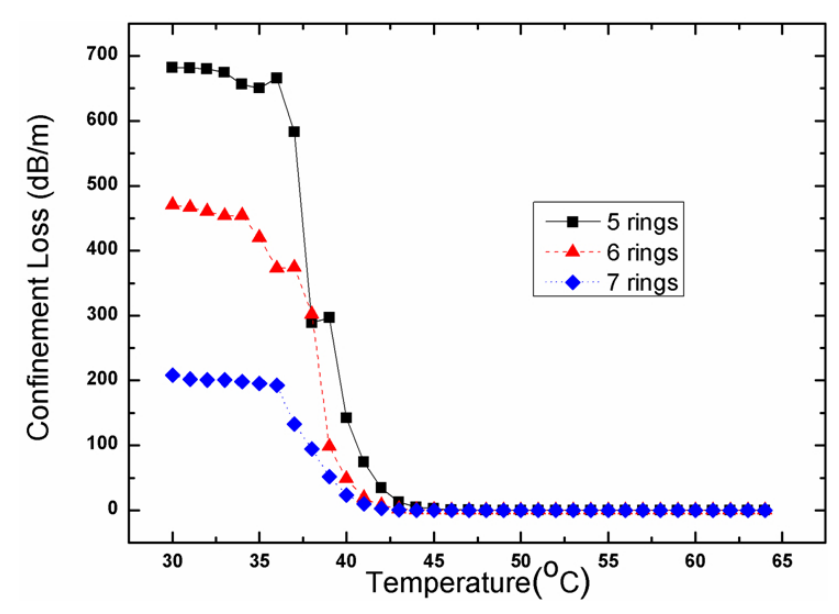

FIG. 3. Confinement loss as functions of temperature with different air-hole rings in the cladding.

filling ratios $d / \Lambda=0.5,0.6,0.7,0.8,0.9$, in the condition that the wavelength is $1550 \mathrm{~nm}$ and there are 6-ring air holes in the cladding. The confinement loss is sensitively dependent on the temperature as we can see from the figure. With the air-filling ratios increases from 0.5 to 0.9 , the high temperature-sensitive range decreases from $15^{\circ} \mathrm{C}$ to $5^{\circ} \mathrm{C}$, and it illustrates that a greater air-filling ratio will enhance the temperature sensitivity of the device. For the smaller air-filling ratio, the propagation properties of MOFs could be developed as an intensity-modulated temperature sensor and for the larger air-filling ratio, the device is promising for a thermo-optic switch or attenuator.

The functional relationship between the confinement loss and the temperature with different air-hole rings in the cladding has been shown in Fig. 3, in the condition that the incident wavelength of $1550 \mathrm{~nm}$ and the air-filling ratio $d / \Lambda=0.8$. The high temperature-sensitive range (from $36^{\circ} \mathrm{C}$ to $40^{\circ} \mathrm{C}$ ) presents independent on the air-hole rings while the confinement loss in the low temperature decreases with

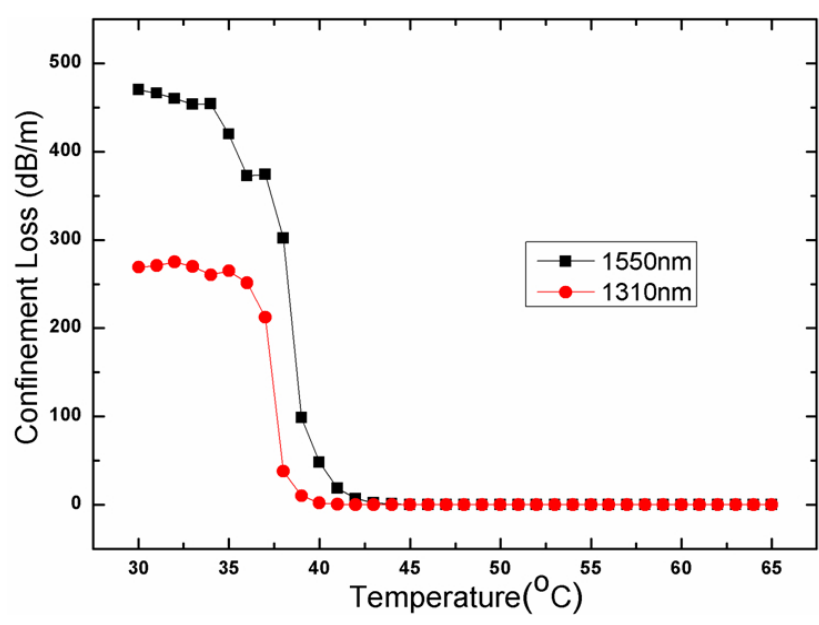

FIG. 4. Confinement loss as functions of temperature with different incident wavelengths.

the air-hole rings, which indicates that the extinction ratio and the temperature sensitivity of the device could be effectively intensified with fewer air-hole rings in the cladding.

Figure 4 shows the confinement loss as functions of temperature at $1310 \mathrm{~nm}$ and $1550 \mathrm{~nm}$ for MOFs with 6-ring cladding air holes and $d / \Lambda=0.8$. It is obvious that there is a stronger relationship between extinction ratio of confinement loss and temperature at $1550 \mathrm{~nm}$ than at $1310 \mathrm{~nm}$. That is to say the temperature dependence is more sensitive for longer wavelength incidence owing to the broadening of the propagation modes of the fibers with increasing wavelength.

\section{EXPERIMENTAL RESULT AND DISCUSSION}

According to the numerical simulation above, the temperature sensitivity of MOFs filled with liquid mixtures of high thermo-optic coefficients presents increasing functional relationship for higher air-filling ratio and longer incident wavelength. Additionally, fewer air-hole rings could enlarge the extinction ratio of the device at the same time. Based on these conclusions, the high air-filling ratio grapefruit MOFs with 1-ring air hole in the cladding supplied by FiberHome Technologies Group are employed in the experimental system and the communication wavelength at $1550 \mathrm{~nm}$ is considered as signal source. As shown in Fig. 5, the microstructured holey region is $87.3 \mathrm{um}$ and the diameter of fiber core is about $12 \mathrm{um}$. The air holes with diameter of $D=34.6$ um are arranged in a hexagonal pattern with the inter-hole distance $\Lambda=35.3 \mathrm{um}$. The fundamental mode of the fiber is guided in the core of the fiber by total internal reflection due to the low effective refractive index of the cladding. The cladding diameter is $125 \mathrm{um}$ as conventional standard single-mode fiber.

The configuration of the experimental setup for the thermooptic characteristic test has been shown in Fig. 6. All of 
the air holes of the grapefruit MOF are infiltrated with mixture liquids of toluene and chloroform simply by capillary action for several tens of seconds. The $4 \mathrm{~cm}$-liquid-filled MOF is then spliced to standard single-mode fibers (SMF) with a splicing loss of $1.5 \mathrm{~dB}$ and placed into the temperature controller in the V-groove of an aluminous slab to avoid bending effects. The transmission powers of mixture liquid-filled MOF at different temperatures are measured as an intensity signal for investigating the temperaturedependent properties. The light source is a tunable semiconductor laser (Agilent 8164A) at the wavelength of $1550 \mathrm{~nm}$. The transmission power is measured by a digital power meter (LX Light Wave) and is recorded from $20^{\circ} \mathrm{C}$ to $60^{\circ} \mathrm{C}$ at $1^{\circ} \mathrm{C}$ intervals. For the correction of the fluctuation of light source, the average values from multiple measurements are operated in the practical experiment, for about 10 times at each temperature.

Figure 7(a) shows both the theoretical confinement loss and the experimental total loss at $1550 \mathrm{~nm}$ for grapefruit MOF as a function of temperature from $25^{\circ} \mathrm{C}$ to $40^{\circ} \mathrm{C}$ with the volume ratio of toluene and chloroform as 7:3. The attenuation of the device varies from $23.3 \mathrm{~dB}$ to $2.6 \mathrm{~dB}$ within the temperature transition range of $5^{\circ} \mathrm{C}$ (from $30^{\circ} \mathrm{C}$ to $34^{\circ} \mathrm{C}$ ), corresponding to the extinction ratio about $20.7 \mathrm{~dB}$ between the low and high transmission states. The obvious transmission jumping phenomenon could be considered as high temperature-sensitive region or optical switching effect. The simulated and experimental results present consistent variation trend. It should be noted that the total loss comprises not only confinement loss but also intrinsic loss

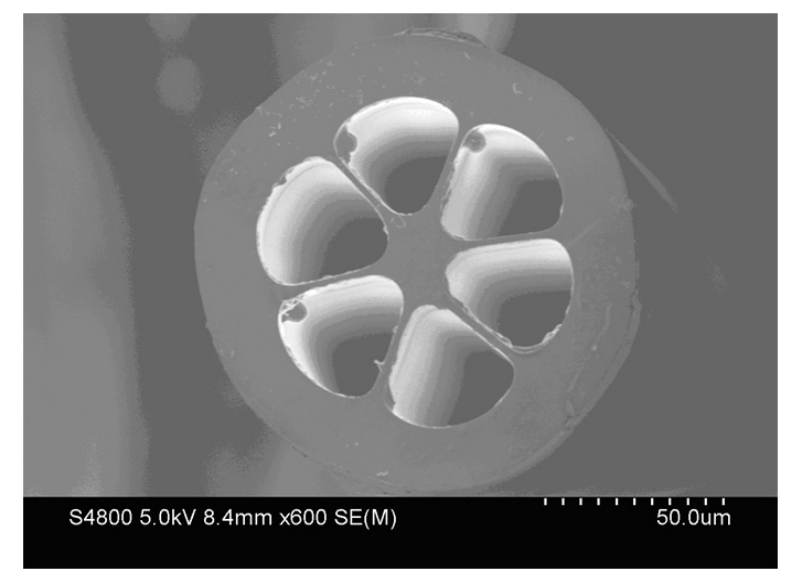

FIG. 5. Optical microscopic images of cross-sections of the grapefruit MOFs.

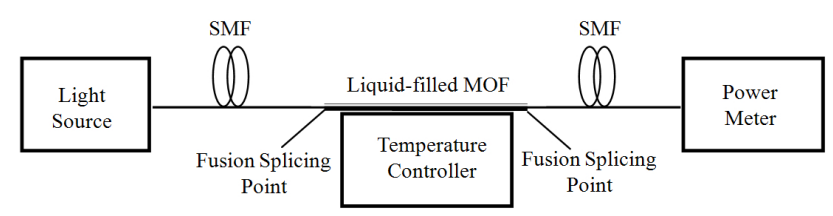

FIG. 6. Scheme of the experimental setup for the thermo-optic characteristic test.

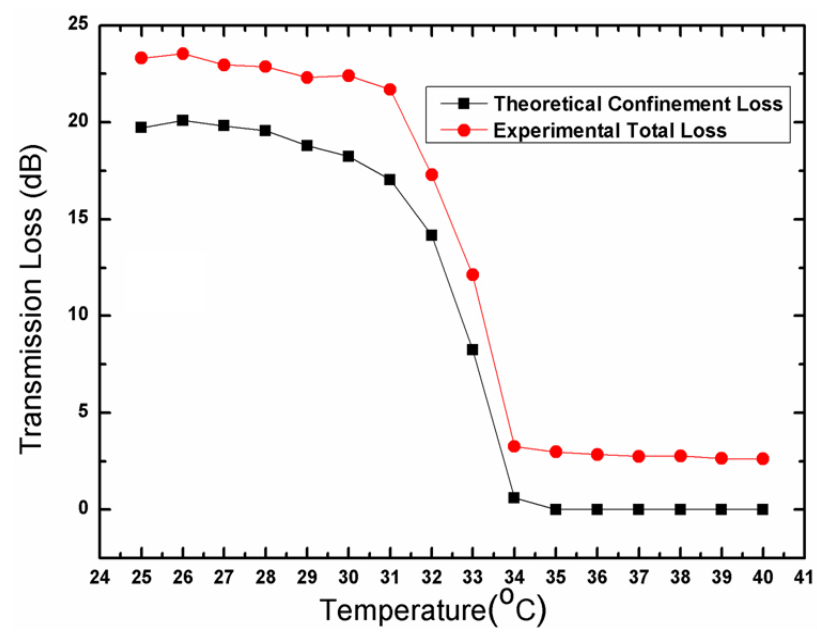

(a)

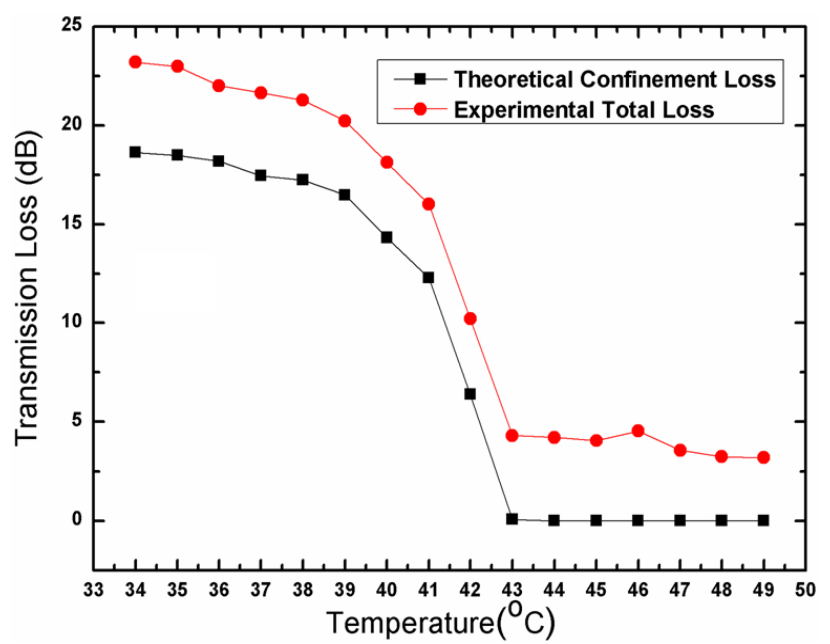

(b)

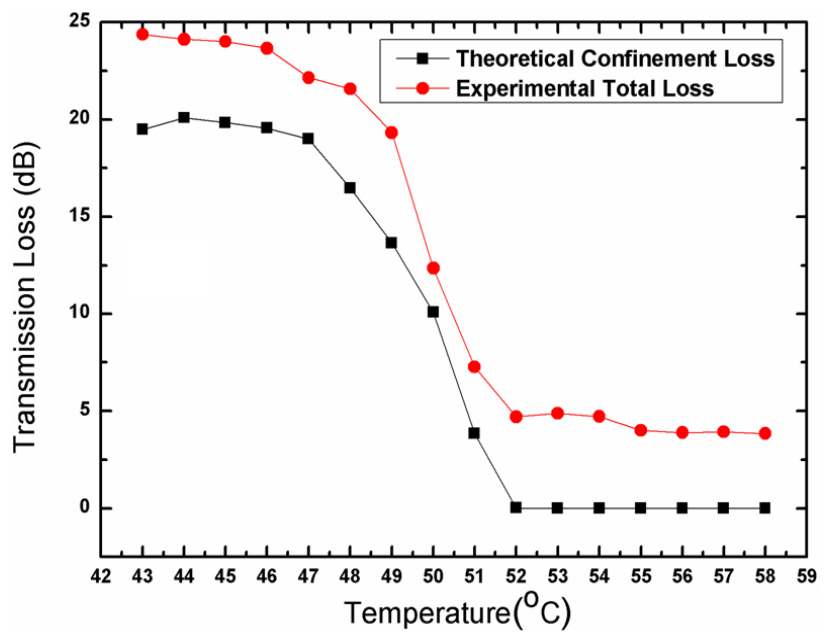

(c)

FIG. 7. Theoretical confinement loss and experimental total loss as functions of temperature. (a) The volume ratio of toluene and chloroform as 7:3. (b) The volume ratio of toluene and chloroform as 8:2. (c) The volume ratio of toluene and chloroform as 9:1. 
and splicing loss, so it is about $3 \mathrm{~dB}$ higher than the theoretical confinement loss.

The physical mechanism of this phenomenon lies in the manipulation of the core mode by the spread of the evanescent mode field, which mainly depends on the distribution of the refractive index of the material infused in the air holes. If the refractive index of the material is lower than that of silica, the mode is confined in the core by total infernal reflection and only a small percentage of the optical field will penetrate into the cladding. In this case, the mode propagates through the fiber with minimal loss. On the other hand, if the refractive index of the material is close to or even higher than that of silica, the mode field will refract into the high index medium, resulting in dramatic loss and attenuation for the propagating mode. As for the practical experiment in this letter, with the temperature decreasing from $40^{\circ} \mathrm{C}$ to $25^{\circ} \mathrm{C}$, the refractive index of liquid-filled cladding gradually increases by $d n / d T \sim 10^{-4} /{ }^{\circ} \mathrm{C}$ according to

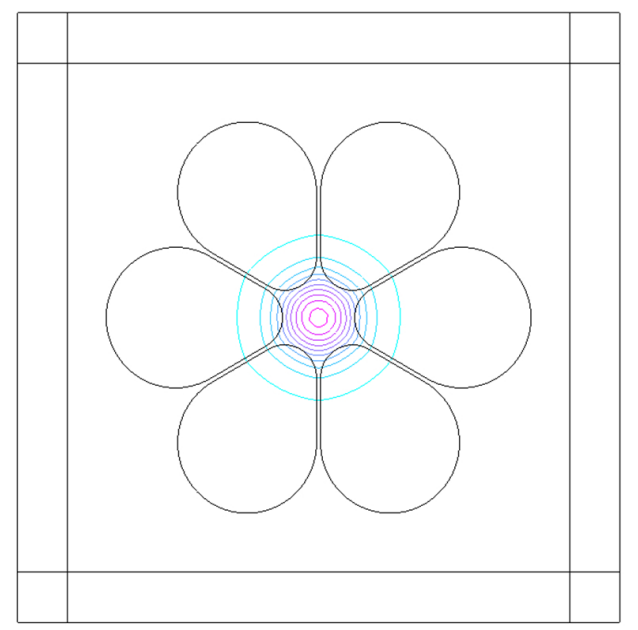

(a)

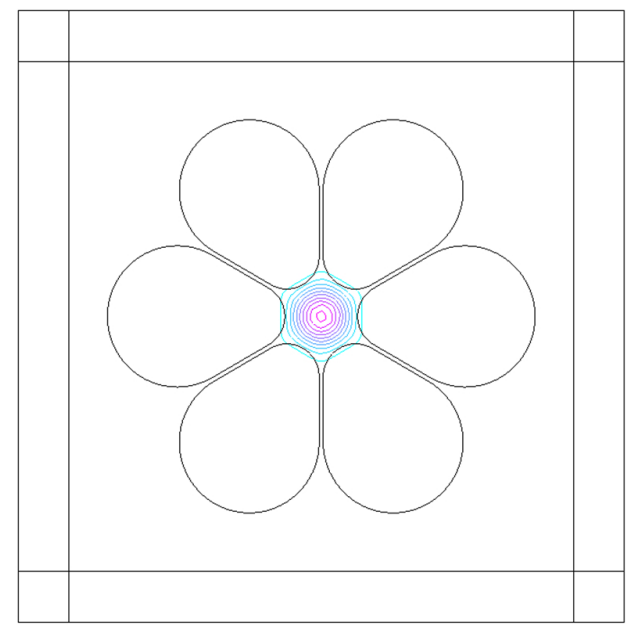

(b)

FIG. 8. The simulated distributions of grapefruit MOF fundamental modes with the volume ratio of toluene and chloroform as $7: 3$. (a) at $30^{\circ} \mathrm{C}$. (b) at $34^{\circ} \mathrm{C}$.
Equation (1), which is equivalent to an lower contrast between the core and cladding indices until the cladding refractive index approximates to that of $\mathrm{SiO}_{2}$ background. The simulated distributions of grapefruit MOF fundamental modes at (a) $30^{\circ} \mathrm{C}$ and at (b) $34^{\circ} \mathrm{C}$ have been plotted in Fig. 8. As seen from the figure, the penetration of the mode field into cladding obviously increases with the decrease of the temperature from $34^{\circ} \mathrm{C}$ to $30^{\circ} \mathrm{C}$, leading to the increment of the confinement loss coefficient of the device.

The similar simulated and experimental results have been shown in Fig. 7(b) and (c) with the volume ratios of toluene and chloroform as 8:2 and 9:1. The insertion losses are 3.5 $\mathrm{dB}$ and $4.1 \mathrm{~dB}$ at higher temperature range while the extinction ratios are about $18.1 \mathrm{~dB}$ and $23.9 \mathrm{~dB}$, respectively. The temperature sensitive ranges are around $40^{\circ} \mathrm{C}$ and $50^{\circ} \mathrm{C}$ within the temperature interval of $5^{\circ} \mathrm{C}$ while the intrinsic loss and splicing loss is about $3 \sim 4 \mathrm{~dB}$. According to the numerical simulation, the high temperature-sensitive range corresponds to the refractive index of the cladding mixture liquids in the numerical interval from 1.454 to 1.457 , which could be achieved through both different proportions of solutions from Equation (2) and functional relationship between temperature and the refractive index of the liquids from Equation (1). That is the physical mechanism for the tunable temperature sensitive range.

The simulation results and the experimental measurements in Fig. 7 present a good qualitative agreement in general with the slight discrepancies arising from the instabilities of liquid fluctuation, the uneven heating of temperature controller and the influence of external environment. Such a temperature tunable device could be developed as a thermo-optic sensor, attenuator or slow response optical switch with a tunable temperature sensitive range. The improvement of the quality of fusion splicing between SMF and MOF will lead to better model confinement and lower insertion loss. Additionally, the effective approaches to increase the extinction ratio lie in the increment of the thermo-optical coefficients and the enhancement of the interaction between the mode field and the infused materials.

\section{CONCLUSION}

In conclusion, we have demonstrated the thermo-optic characteristics of liquid-filling micro-structured optical fiber both theoretically and experimentally. Numerical simulation for the optimization of its structure has been operated and discussed in the aspects of the air-filling ratios $d / \Lambda$, the incident wavelength and the number of air rings in the cladding. For the optimum grapefruit MOF, it presents a tunable temperature-sensitive range according to the concentration of mixture liquids. Such a device presents an insertion loss of $3 \sim 4 \mathrm{~dB}$ and an extinction ratio of $\sim 20 \mathrm{~dB}$ within the narrow temperature interval of $5^{\circ} \mathrm{C}$. With the appropriate standardization of the intensity of the transmission signal, 
the proposed liquid-filling MOF could be exploited as an intensity-modulated high sensitivity temperature sensor in the region of optical fiber sensing. In addition, as for the optical communication system, the MOF could be developed as all-in-fiber photonic functional devices (attenuator or optical switch) with the merits of simple structure, compact configuration and easy fabrication.

\section{ACKNOWLEDGMENT}

This work was supported by the National Key Basic Research and Development Program of China (Grant No. 2010CB327801), the National Natural Science Foundation of China (Grant No. 11204212), and China Postdoctoral Science Foundation Funded Project (Grant No. 2012M520024).

\section{REFERENCES}

1. J. C. Knight, "Photonic crystal fibres," Nature 424, 847-851 (2003).

2. P. Russell, "Photonic crystal fibers," Science 299, 358-362 (2003).

3. J. C. Knight, T. Birks, P. Russell, and D. Atkin, "All-silica single-mode optical fiber with photonic crystal cladding," Opt. Lett. 21, 1547-1549 (1996).

4. J. C. Knight, J. Broeng, T. Birks, and P. Russell, "Photonic band gap guidance in optical fibers," Science 282, 14761478 (1998).

5. W. J. Lee, D. C. Kim, S. G. Park, E. H. Lee, and S. G. Lee, "Measurement of the internal structure of an optical waveguide embedded in a flexible optical circuit board by enhancing the signal contrast of a confocal microscope," J. Opt. Soc. Korea 15, 9-14 (2011).

6. M. Bozorgi and N. Granpayeh, "Directional emission from photonic crystal waveguide output by terminating with CROW and employing the PSO algorithm," J. Opt. Soc. Korea 15, 187-195 (2011).
7. B. Eggleton, C. Kerbage, P. Westbrook, R. Windeler, and A. Hale, "Microstructured optical fiber devices," Opt. Express 9, 698-713 (2001).

8. R. He, P. Sazio, A. Peacock, N. Healy, J. Sparks, M. Krishnamurthi, V. Gopalan, and J. Badding, "Integration of gigahertz-bandwidth semiconductor devices inside microstructured optical fibres," Nat. Photonics 6, 174-179 (2012).

9. M. Danaie and H. Kaatuzian, "Bandwidth Improvement for a photonic crystal optical Y-splitter," J. Opt. Soc. Korea 15, 283-288 (2011).

10. C. S. Park, K. I. Joo, S. W. Kang, and H. R. Kim, "A PDMS-coated optical fiber Bragg grating sensor for enhancing temperature sensitivity," J. Opt. Soc. Korea 15, 329-334 (2011).

11. T. Larsen, A. Bjarklev, D. Hermann, and J. Broeng, "Optical devices based on liquid crystal photonic bandgap fibres," Opt. Express 11, 2589-2596 (2003).

12. Y. Yu, X. Li, X. Hong, Y. Deng, K. Song, Y. Geng, H. Wei, and W. Tong, "Some features of the photonic crystal fiber temperature sensor with liquid ethanol filling," Opt. Express 18, 15383-15388 (2010).

13. Y. Wang, W. Jin, L. Jin, X. Tan, H. Bartelt, W. Ecke, K. Moerl, K. Schroeder, R. Spittel, and R. Willsch, "Optical switch based on a fluid-filled photonic crystal fiber Bragg grating," Opt. Lett. 34, 3683-3685 (2009).

14. Y. Wang, X. Tan, W. Jin, D. Ying, Y. Hoo, and S. Liu, "Temperature-controlled transformation in fiber types of fluid-filled photonic crystal fibers and applications," Opt. Lett. 35, 88-90 (2010).

15. Y. Wang, H. Bartelt, W. Ecke, K. Moerl, H. Lehmann, K. Schroeder, R. Willsch, J. Kobelke, M. Rothhardt, and R. Spittel, "Thermo-optic switching effect based on fluid-filled photonic crystal fiber," IEEE Photon. Technol. Lett. 22, 164-166 (2010).

16. A. Samoc, "Dispersion of refractive properties of solvents: chloroform, toluene, benzene, and carbon disulfide in ultraviolet, visible, and near-infrared," J. Appl. Phys. 94, 6167-6174 (2003).

17. W. Heller, "Remarks on refractive index mixture rules," J. Phys. Chem. 69, 1123-1129 (1964). 